\title{
PENGILS OF POLARITIES IN PROJECTIVE SPACE
}

\author{
SEYMOUR SCHUSTER
}

1. Introduction. A polarity in complex projective space of two dimensions $\left(S_{2}\right)$ is completely determined by a self-polar triangle $A B C$, and a pair of corresponding elements: a point $P$ and its polar line $p$. We denote the polarity by $(A B C)(P p)$. We follow Coxeter (2) in defining a pencil of polarities as the $\infty^{1}$ polarities $(A B C)(P p)$ where $A, B, C, P$ are fixed while $p$ varies in a pencil of lines. Coxeter has developed this notion and has shown that two of the classical types of pencils of conics are, in fact, the conics which arise from such pencils of polarities. The pencils of conics so derived are the general system with four distinct points in common and the double-contact system where the conics have in common two distinct points and common tangents at these points.

It is well known that a polarity in $S_{2}$ is also completely determined by a self-polar pentagon (2, p. 64). Suppose we start afresh and define a pencil of polarities by means of such a pentagon. Will this yield anything more general than the previous definition? The answer to this question is in the affirmative, as we shall see in $\S 2$.

Sections 3 and 4 deal with a development of pencils of polarities in complex projective space of three dimensions $\left(S_{3}\right)$ by utilizing a self-polar tetrahedron in the definition. Section 5 continues the development in $S_{3}$, but by redefining a pencil of polarities, this time using a self-polar skew hexagon. Section 6 will deal with the problem in projective space of $n$ dimensions $\left(S_{n}\right)$.

\section{Pentagonal pencils of polarities.}

Definition. A pentagonal pencil of polarities is the set of polarities determined by the self-polar pentagon $P Q R S T$, where $P, Q$, and $T$ remain fixed and the line $R S$ varies in a pencil of lines with $R$ on the fixed line $t$ through $Q$, and $S$ on the fixed line $q$ through $T$ (Fig. 1).

Each polarity for such a pentagon has

$$
P \leftrightarrow p=R S, Q \leftrightarrow q=S T, R \leftrightarrow r=T P, S \leftrightarrow s=P Q, T \leftrightarrow t=Q R .
$$

If we consider a general pentagon (with none of the vertices on its own polar), we can find a fixed self-polar triangle for the pencil. Let $A$ denote the point of intersection $q \cdot t$. Then $A \leftrightarrow a=Q T$, and $A Q T$ is a fixed self-polar triangle for the entire pencil. If the pencil of lines $p$ varies about a fixed point on a side of $A Q T$, we can arrive at a self-dual system $(2,5.82)$, as well as the more general type of pencil when $p$ varies about a point in general position.

Received April 16, 1955. The author wishes to express appreciation to Professor H. S. M. Coxeter for his aid and encouragement. 


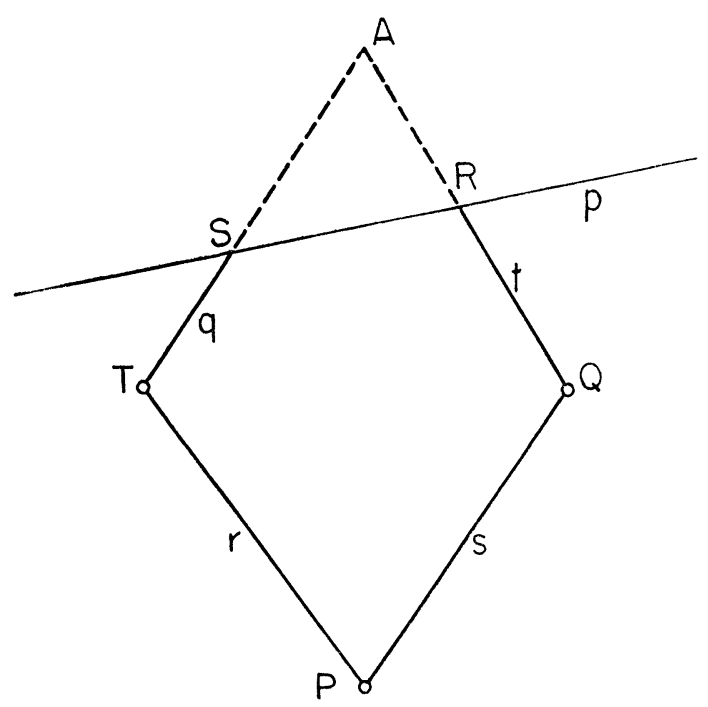

Figure 1

Hence, a pentagonal pencil yields everything which can be found by a development with a fixed self-polar triangle.

We are particularly interested in finding pentagonal pencils of polarities which have no fixed self-polar triangle. Recalling the construction for a selfpolar triangle in a polarity determined by a self-polar pentagon, we see that two alternate vertices of the pentagon must be joined. Now, if there is a pencil of polarities determined by pentagon $P Q R S T$ with $P, Q, T$ fixed and the line $R S$ varying in a pencil, the only possible fixed self-polar triangle is $Q T(Q R \cdot S T)$; for, no pair of alternate vertices other than $Q$ and $T$ yield a fixed line. If $Q T$ is distinct from $S T=q$, such a triangle is non-degenerate. Hence, attention will be confined to pentagons where $Q T$ coincides with $S T$. That is, $Q$ is always on its own polar $q$.

We can immediately state two theorems of (2) which carry over to the case where $Q$ is always a self-conjugate point. Their proofs, which we omit, are very much the same as those supplied in (2), except that there are several additional special cases to consider.

THeOREm 2.1. The polars of any fixed point $X$, with respect to a pencil of polarities, form a pencil of lines through a point $X^{\prime}$.

Theorem 2.2. The locus of $X^{\prime}$ as $X$ varies along a fixed line $o$ is a conic or a line. The locus of poles of the fixed line o, with respect to a pencil of polarities is the same conic or line.

Suppose, for instance, that $P^{\prime}$, the vertex of the pencil of lines $p$, is located on $r$. Then all the polarities in the pencil induce on $r$ the same involution, namely $\left(P P^{\prime}\right)\left(T T_{1}\right)$, where $T_{1}$ is $t \cdot r$. The invariant points, $A$ and $B$, of this involution, must be common points of the conics determined by the polarities. 
Thus we have a pencil of conics with a common tangent $q$ at $Q$ and two further common points, $A$ and $B$.

It might be best to introduce some general notation at this point. We will discuss pencils of polarities in $i$-dimensional space, determined by a configuration generally associated with $j$ points. We denote such a pencil of polarities by the symbol $P^{i}{ }_{j k}$, where $k$ will be an integer indicating a special case of such a pencil. The cases covered by Coxeter were 2 -dimensional, defined by triangles; so we call them the $P^{2}{ }_{31}$ and $P^{2}{ }_{32}$ systems. The $P^{2}{ }_{31}$ system is the one yielding a general pencil of conics with four distinct points in common. The $P^{2}{ }_{32}$ system is Coxeter's self-dual system yielding a double-contact pencil of conics. Our pentagonal pencils have yielded $P^{2}{ }_{31}$ and $P^{2}{ }_{32}$, so we will not trouble to give them alternate names $P^{2}{ }_{5 k}$. Instead, we begin naming pentagonal systems with the pencil discussed above, which yielded a new pencil of conics. We call this a $P^{2}{ }_{51}$ system.

Suppose we turn our problem around and ask how to determine the pentagon for the pencil of polarities when we are given the pencil of conics with a common tangent $q$ at $Q$, and two further points $A$ and $B$ in common. The following procedure yields the pentagon. Let $A B=r$ and $T=r \cdot q$ (Fig. 2). Find $T_{1}$ on $r$ such that $\mathrm{H}\left(T T_{1}, A B\right)$. Let $t=Q T_{1}$. Line $t$ is the polar of $T$ with respect to the pencil of conics.

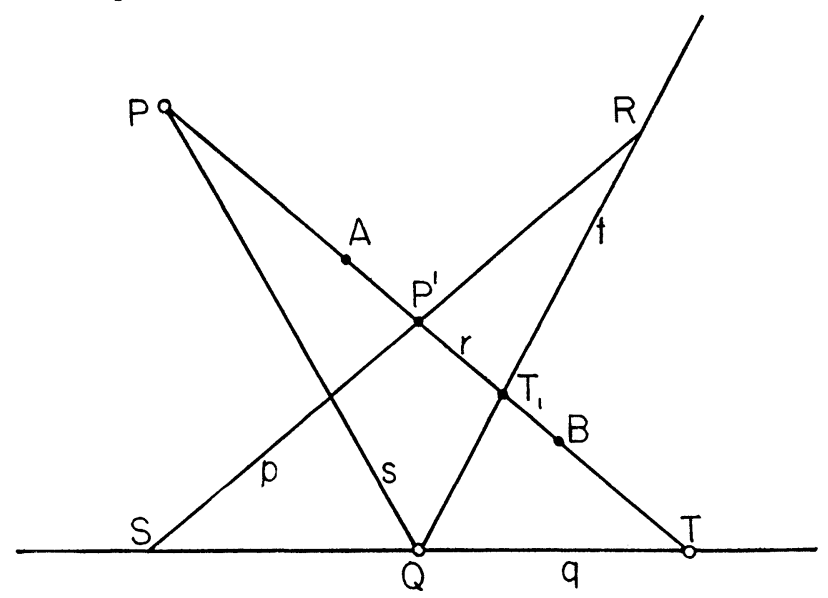

Figure 2

Pick a point $P$ arbitrarily on $r$. Let $s=P Q$. Now two cases arise:

(a) $P \neq A, B$. Find $P^{\prime}$ on $r$ such that $\mathrm{H}\left(P P^{\prime}, A B\right)$. An arbitrary line through $P^{\prime}$ will serve to represent the variable line $p$. Thus we have a self-polar pentagon $P Q R S T$, where $p \cdot t=R$ and $p \cdot q=S$, which determines the pencil of conics provided $p$ varies in the pencil about $P^{\prime}$.

(b) $P=A$. The point $P$ is self-conjugate. Construct an arbitrary line through $P$ to serve as a representative for the variable pencil. Each arbitrary $p$ is a tangent at $P$ for one conic of the pencil. This time $P$ plays the role of $P^{\prime}$ so 
we have a pentagon which defines the pencil of polarities associated with the conics provided line $p$ varies in the pencil through $P$.

Suppose we allow the pencil of lines $p$ to have its vertex on $q$, so that $P^{\prime}=S$. We will show that such a pencil of polarities leads to a pencil of conics with four-point contact (third order) at point $Q$. We shall call these polarities a $P^{2}{ }_{52}$ system. In order to prove our assertion we show that every point on line $q$ possesses the same polar for every polarity in the pencil (10, p. 136). From the nature of the pentagon defining the pencil, it is seen that $Q, S$, and $T$ have fixed polars for the pencil. For a consideration of the general point $X$ on $q$, we look back at the construction of its polar line $x$ (see Fig. 3 ). It is clear that $x$ depends not at all upon the position of $p$, except for the fact that $p$ meets $q$ in $S$. But this is so for every polarity in the pencil. Hence, point $X$ has a fixed polar line for the entire pencil.

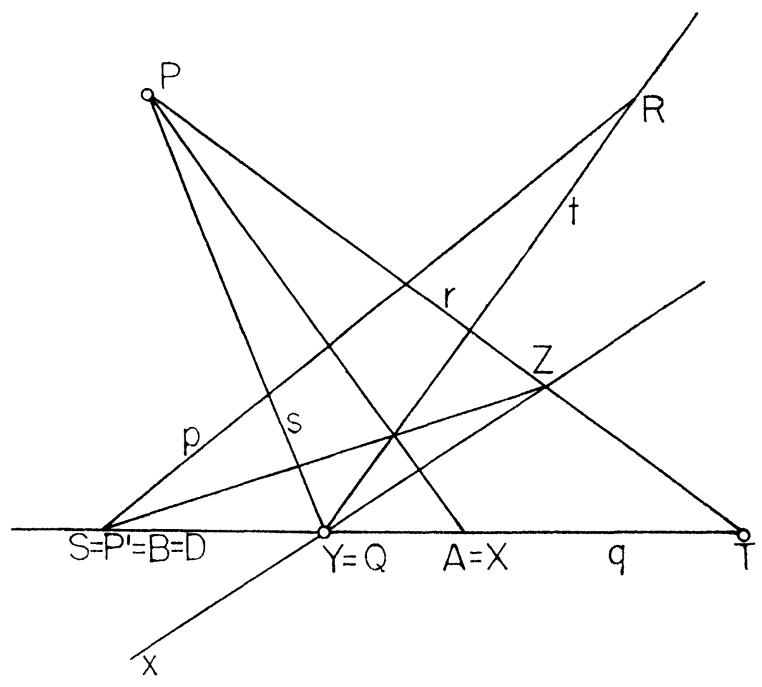

Figure 3

N.B. The only way such a pencil of conics can be derived from a pentagonal pencil of polarities is by having point $S$ fixed. For, otherwise, there would be points on $q$ which would not have the same polar for all polarities in the pencil, thus contradicting the definition (10, p. 136).

The fact that a four-point contact system of conics is reached by having $p$ rotate about a fixed $S$ can be seen in a simpler and perhaps more elegant manner as follows:

$X \pi x$ is a projectivity of points $X$ on $q$ with their polars through $Q$. Suppose point $X$ had two distinct polars for two different polarities in the pencil. Then $Q S T X \pi q s t x_{1}$ and $Q S T X \pi q s t x_{2}$. But this contradicts the fundamental theorem of projective geometry. Therefore $x_{1}=x_{2}$.

Actually, the $P^{2}{ }_{51}$ system may be reached with fewer restrictions than we have placed on a pentagonal pencil of polarities. That is, line $p$ may vary 
about point $P^{\prime}$, where $P^{\prime}$ is located anywhere except upon line $q$. We have already discussed the case where $P^{\prime}$ is on $r$. We will now discuss the case where $P^{\prime}$ is in a general position; the reader might supply the details of the development in the cases where $P^{\prime}$ is on line $t$, line $s$, or even when $P^{\prime}=P$. The same system of conics is reached in all.

$P^{\prime}$ is in general position. Since $Q$ is on $q$, every polarity in the pencil yields a conic with $q$ as the tangent at point $Q$. The pencil of conics cannot have three-point contact (second order) at $Q$ because point $T$ on $q$ has the same polar for all the polarities, thus contradicting the necessary conditions for three-point contact (10, p. 134). The conics cannot have four-point contact, either, owing to the observation on p. 122. Therefore, the pencil must have two-point contact at $Q$, and two other points $A$ and $B$ in common. Since $T$ and its polar $t$ are fixed for the pencil, $A$ and $B$ must be collinear with $T$ such that $\mathrm{H}\left(A B, T T_{1}\right)$, where $T_{1}=t \cdot A T$.

Examination of the special cases where $P^{\prime}$ is on $s$, on $t$ or $P^{\prime}=P$, leads to an actual determination of the points $A$ and $B$, whereas our examination of the more general case merely established the existence of $A$ and $B$.

Concerning pencils of conics with three-point contact at $Q$, we might say that all cases of pentagonal pencils have been investigated and no such systems of conics result; hence, they cannot be derived from a pentagonal pencil of polarities. However, there are more direct methods which are far more convincing:

(1) A pencil with three-point contact (second order) has $Q$ as the only point on the tangent $q$ which possesses the same polar for all polarities in the pencil (10, p. 134). But, this is always contradicted by point $T$, whose polar line is fixed for the pencil.

(2) A pencil of conics with three-point contact at $Q$ has another point, say $A$, in common. Suppose that such a system of conics arises from a pentagonal pencil. Let $T_{1}=t \cdot A T$. The point $B$, the harmonic conjugate of $A$ with respect to $T$ and $T_{1}$, is also common to all the conics. This contradicts the hypothesis that a three-point contact system arises from a pentagonal pencil.

Summary. The investigation of pentagonal pencils of polarities has shown them to be more general than the triangular pencils. The triangular pencils $P^{2}{ }_{31}$ and $P^{2}{ }_{32}$ can both be derived as pentagonal pencils. Further, two new systems, $P^{2}{ }_{51}$ and $P^{2}{ }_{52}$, were found to yield pencils of conics: (1) with two-point contact at a point and two other distinct points in common, and (2) the four-point contact system. It was also shown that the remaining pencil of conics, with three-point contact, cannot be derived from pentagonal pencils.

\section{Tetrahedral pencils of polarities in $S_{3}$.}

Definition. A tetrahedral pencil of polarities is the set of polarities $(A B C D)(P \pi)$, where $A, B, C, D$, and $P$ are all fixed while plane $\pi$ varies in an axial pencil. 
THEOREM 3.1. The polar planes of any fixed point $X$, with respect to a pencil of polarities, form a pencil of planes.

Proof. We use the following construction (4) for the polar plane of an arbitrary point. Let the polarity be $(A B C D)(P \pi)$, and $X$ a point in general position. We seek $\chi$, the polar plane of $X$. We use the notation

$$
A B C=\delta, B C D=\alpha, A C D=\beta, A B D=\gamma,
$$

$D P \cdot \delta=P_{\delta}, D X \cdot \delta=X_{\delta}, \pi \cdot \delta=p_{\delta}$ (Fig. 4). Since $D P$ is the polar line of $p_{\delta}$, the polarity induced in plane $\delta$ is $(A B C)\left(P_{\delta} p_{\delta}\right)$. By Coxeter's construction $(2,5.64)$, the polar of $X_{\delta}$ in plane $\delta$ is line

$$
x_{\delta}=\left[A P_{\delta} \cdot\left(a \cdot P_{\delta} X_{\delta}\right)\left(p_{\delta} \cdot A X_{\delta}\right)\right] \cdot\left[B P_{\delta} \cdot\left(b \cdot P_{\delta} X_{\delta}\right)\left(p_{\delta} \cdot B X_{\delta}\right)\right] .
$$

By performing a similar construction in plane $\alpha$, we can get the polar $x_{\alpha}$ of the point $X_{\alpha}$. The plane determined by $x_{\alpha} x_{\delta}$ is the polar plane of point $X$. In order to prove this it is sufficient to remark that $X$ is conjugate to every point on $x_{\alpha}$ and to every point on $x_{\delta}$.

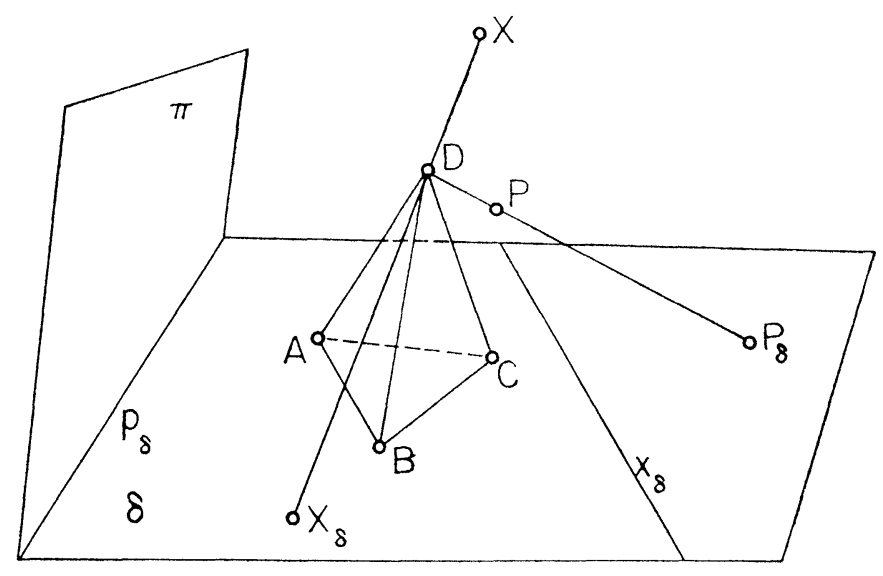

FIGURE 4

Referring to the construction, we proceed.with the proof. Let $\pi$ vary in a pencil about line $p^{\prime}$. This line cannot lie in more than two of the four faces. Let $\alpha$ and $\delta$ be two in which it does not lie. Then $p^{\prime} \neq p_{\delta}$. Line $p_{\delta}$ varies in a pencil (in plane $\delta$ ) about the point $P^{\prime}$. By Coxeter's result $(2,5.81$ ), we know that line $x_{\delta}$ likewise varies in a pencil (in plane $\delta$ ) about a point $X^{\prime}{ }_{\delta}$. Similarly, line $x_{\alpha}$ varies in a pencil (in plane $\alpha$ ) about a point $X_{\alpha}^{\prime}$. In each polarity of the pencil we have $\chi$, the polar plane of $X$, determined by $x_{\alpha} x_{\delta} . \quad \chi$ must pass through $X^{\prime}{ }_{\alpha}$ and $X^{\prime}{ }_{\delta}$ in each of the polarities. Hence, the polar planes of $X$ vary in a pencil about $X_{\alpha}^{\prime} X^{\prime}{ }_{\delta}$.

In case $X_{\alpha}^{\prime}=X^{\prime}{ }_{\delta}$, we take $X^{\prime}{ }_{\beta}$ and $X^{\prime}{ }_{\gamma}$. All four cannot coincide. 
Three special cases of tetrahedral pencils are of interest because they lead to systems, highly analogous to the self-dual systems discussed in (2). These arise when line $p^{\prime}$ meets one or more edges of $A B C D$.

There will be four tetrahedral pencils $P^{3}{ }_{4 k}$, where $k$ is $0,1,2$, or 3 , according as line $p^{\prime}$ meets $0,1,2$ or 3 edges of $A B C D$. It is clear that if $p^{\prime}$ were to meet more than three edges of the self-polar tetrahedron we would not have a welldefined pencil.

The $P^{3}{ }_{41}$ system. Suppose $p^{\prime}$ meets only one edge, say $A B$, of the self-polar tetrahedron. Then $P^{\prime}{ }_{\delta}=P^{\prime}{ }_{\gamma}$ is on $A B$. From the discussion in (2) we know that the polarities induced in the two faces through $A B$ are two-dimensional selfdual systems $P^{2}{ }_{32}$. Any pair of the involution on $A B$, together with $C$ and $D$, will give a fixed self-polar tetrahedron for the entire pencil. Therefore, we have not one, but $\infty^{1}$ tetrahedra which are self-polar for all polarities in the pencil.

TheOREM 3.2. If two polarities belong to a $P^{3}{ }_{41}$ system, their product is a general axial homography (1, p. 385; 5, pp. 180-183).

Proof. Let $p^{\prime}$ meet $A B$ in $P^{\prime}{ }_{\delta}$. A self-dual system is induced in plane $A B C$. Therefore, the product of two polarities in $P^{3}{ }_{41}$ leaves invariant every point on $A B$. Also, points $C$ and $D$ are invariant, so we must have the pencil of planes with axis $C D$ as an invariant pencil. Thus $C D$ is the tangential-axis and $A B$ is the point-axis of a general axial homography; $C$ and $D$ are the invariant points.

The $P_{42}^{3}$ system. We suppose now that $p^{\prime}$ meets exactly two edges, say $A B$ and $C D$, of the self-polar tetrahedron. This implies that pencils of polarities induced in the faces of $A B C D$ are all self-dual, with the same involutions of points on $A B$ and $C D$ for all the polarities in the pencil. Therefore, if we take any pair of the involution on $A B$ together with any pair of the involution on $C D$, we get four points which determine a self-polar tetrahedron for the entire pencil. Hence, $\infty^{2}$ self-polar tetrahedra are available in a $P^{3}{ }_{42}$ system.

THEOREM 3.3. If two polarities belong to a $P^{3}{ }_{42}$ system, their product is a hyperbolic biaxial homography.

Proof. Lines $A B$ and $C D$ are pointwise invariant under the product. Since a hyperbolic biaxial homography is characterized by two such lines, the theorem is proved.

The $P^{3}{ }_{43}$ system. Suppose $p^{\prime}$ meets three edges of $A B C D$. It must lie in a face, say plane $\delta$. The polarity induced in $\delta$ is, therefore, the same for each member of the pencil. The polarity is always $(A B C)\left(P_{\delta} p^{\prime}\right)$. Hence, the polar planes of $X$ (for all members of the pencil) pass through line $x_{\delta}$. The involutions of conjugate points induced on $A B, B C$, and $A C$ are the same for all members of the pencil. By taking any pair of the involution on one of these lines together with the opposite vertex (the pole of the line in plane $\delta$ ), we can arrive at an infinite number of self-polar triangles. In fact, there are $\infty^{3}$ such. Therefore, 
we have $\infty^{3}$ tetrahedra (formed by $D$ and the $\infty^{3}$ self-polar triangles in plane $\delta$ ) which are self-polar for all the polarities. Hence

THEOREM 3.4. $A P^{3}{ }_{43}$ system admits a plane $\delta$ upon which the induced polarity is the same for all members of the pencil. The polars of any point $P$ pass through a fixed line $p^{\prime}$ on $\delta$, and the poles of any plane $\pi$ form a range of points on a fixed line $D P_{\delta}$, where $D$ is the pole of $\delta$ for all the polarities.

(If we define a range of polarities by fixing tetrahedron $A B C D$ and plane $\pi$, while $P$, the pole of $\pi$, varies in a range, then the above theorem indicates that a $P^{3}{ }_{43}$ system is also a range of polarities. Therefore, we might call it a self-dual system in $S_{3}$.)

THEOREM 3.5. If two polarities belong to the same $P^{3}{ }_{43}$ system, their product is a spatial homology.

Proof. In a $P^{3}{ }_{43}$ system every line through one vertex, say $D$, of the selfpolar tetrahedron is invariant, and every pencil of planes whose axis lies in $\delta$ is invariant. But we must show that every point of a plane is invariant and every plane through a point is invariant $(\mathbf{1 0}$, p. $75 ; \mathbf{5}$, p. 179). Consider any point $X$ in $\delta$. Its polar plane $\chi$ meets $\delta$ in line $x^{\prime}$. If we operate with another member of the self-dual system, we find that $x^{\prime} \rightarrow X$ (because the polarity induced in $\delta$ is the same for all members of the system). Hence, $X \rightarrow X$, which shows that every point of $\delta$ is invariant. Now consider an arbitrary plane $\rho$ through $D$. Let $\rho \cdot \delta=r^{\prime}$ and let $R^{\prime}$ be the pole of $r^{\prime}$ in $\delta$. Then $R$, the pole of $\rho$, is on line $D R^{\prime}$. Then operate on $R$ with another member of the pencil. The construction tells us that $R$ maps into a plane through $r^{\prime}$. Since every line through $D$ is invariant, the plane $r^{\prime} D$ is invariant. Hence we have a spatial homology with $D$ as its center and $\delta$ its plane of perspectivity.

THEOREM 3.6. Every spatial homology can be expressed as the product of two polarities belonging to a self-dual system $\left(P^{3}{ }_{43}\right)$.

Proof. The homology whose plane of perspectivity is $\delta$ and whose center is $D$, with a pair of related points $P_{1}$ and $P_{2}$ (on a line through $D$ ) (10, p. $76 ; 5$, p. 179), is the product of polarities $(A B C D)\left(P_{1} \pi\right)$ and $(A B C D)$ $\left(P_{2} \pi\right)$, where $\pi$ is an arbitrary plane.

THEOREM 3.7. The locus of poles of a fixed plane with respect to a pencil of polarities is:

(a) a twisted cubic passing through the vertices of the fixed self-polar tetrahedron in a $P_{40}^{3}$ pencil.

(b) a conic passing through three vertices of one of the fixed self-polar tetrahedra in a $P^{3}{ }_{41}$ pencil.

(c) a line passing through two of the vertices of one of the fixed self-polar tetrahedra in a $P^{3}{ }_{42}$ pencil.

(d) a line passing through the vertex common to all the self-polar tetrahedra in a $P_{43}^{3}$ pencil. 
Proof.

(d) For a self-dual system $\left(P_{43}^{3}\right)$, Theorem 3.4 indicates that the locus is such a line.

(a) We consider the general case $P^{3}{ }_{40}$ with the pencil defined by $(A B C D)(P \pi)$. Let $\chi$ be a fixed plane. Take three points $Q_{1}, Q_{2}$, and $Q_{3}$ on $\chi$. The intersection of the polar planes of these three points is the pole $X$ of $\chi$. Theorem 3.1 indicates that as $\pi$ varies in the pencil the polar planes of $Q_{1}, Q_{2}, Q_{3}$ vary in projective axial pencils. The locus of poles is, therefore, a twisted cubic.

Consider this situation from another point of view (Fig. 5). Examining what happens in plane $\delta$, we see that line $x_{\delta}$ is fixed. The locus of poles of $x_{\delta}$ in $\delta$ is a conic through $A, B$, and $C(2,6.81)$. This conic is the projection of the cubic onto $\delta$ from point $D$, the pole of $\delta$. Hence, the cubic must be embedded in the surface of a quadric cone with vertex $D$. Further the cubic actually passes through $D$; for otherwise the projection of the curve onto the plane would be of third order. Similarly, the cubic passes through $A, B$ and $C$.

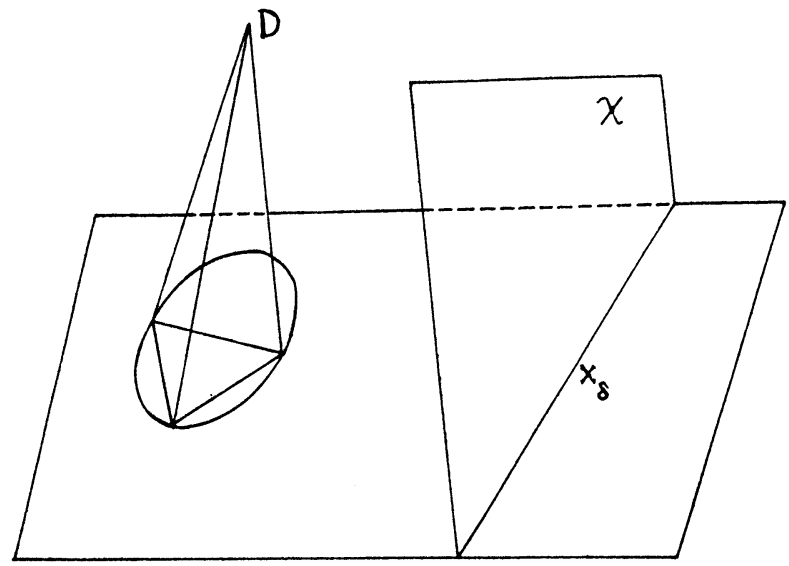

Figure 5

(b) (Fig. 6) In a $P^{3}{ }_{41}$ system, self-dual systems $\left(P^{2}{ }_{32}\right)$ are induced in two faces which have line, say $A B$, in common. By the nature of such self-dual systems, we see that point $X_{\delta}$, the projection of $X$ through $D$ onto plane $\delta$, describes a line through $C$. Likewise, in plane $\gamma$, the point $X_{\gamma}$ describes a line through $D$, so that the locus of poles of $\chi$ is confined to plane $D C A_{\delta}$, where $A_{\delta}$ is the companion of $X^{\prime}{ }_{\delta}=\chi \cdot A B$ in the involution on line $A B$. Further, the projection of the locus through $B$ onto $\beta$ is a conic; and the projection through $A$ onto $\alpha$ is also a conic. Hence, the locus of $X$ is a conic through $C, D$, and $A_{\delta}$, where $C, D$, and $A_{\delta}$ form the vertices of a self-polar tetrahedron for the entire pencil.

(c) (Fig. 7) In a $P^{3}{ }_{42}$ system, self-dual systems $\left(P^{2}{ }_{32}\right)$ are induced in all the faces of $A B C D$. Let $\chi \cdot A B=X^{\prime}{ }_{\delta}$ and $\chi \cdot C D=X^{\prime}{ }_{\alpha}$. Let $A_{\alpha}$ be the companion 


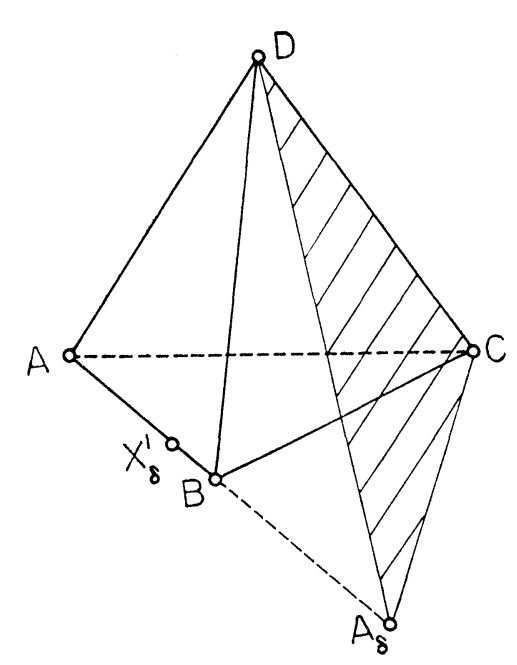

Figure 6

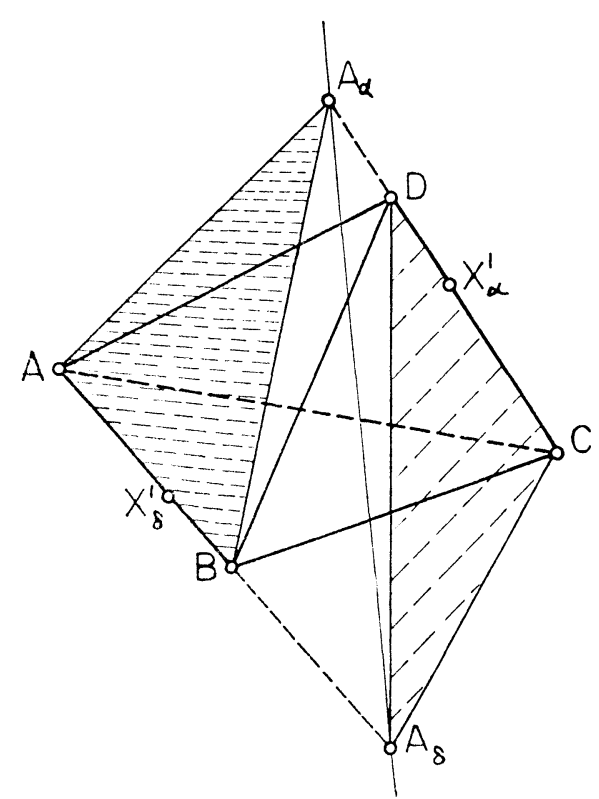

FIGURE 7

of $X^{\prime}{ }_{\alpha}$ in the involution on $A B$, and let $A_{\alpha}$ be the companion of $X^{\prime}{ }_{\alpha}$ in the involution on $C D$. The projection of $X$ onto $\delta$ is the line $C A_{\delta}$; onto plane $\gamma$, the line $D A_{\delta}$; and on to plane $\beta$, the line $A A_{\alpha}$. Therefore, the locus of $X$ must be on planes $C D A_{\delta}$ and $A B A_{\alpha}$. The locus is, in fact, the meet of these planes, that is, the line $A_{\alpha} A_{\delta}$ The points $A_{\alpha}, A_{\delta}, X^{\prime}{ }_{\alpha}$, and $X^{\prime}{ }_{\delta}$ form one of the ${ }^{2}$ tetrahedra which are self-polar for the entire pencil.

From previous discussion we know that the polar plane of a fixed point $P$ varies in an axial pencil. We call the axis of the pencil the axis of point $P$ and denote it by $l_{P}$. It might be noted that there are $\infty^{3}$ axes $l_{P}$ since there are $\infty^{3}$ points in $S_{3}$. But there are $\infty^{4}$ lines in $S_{3}$, so we may conclude that not every line is an axis of a point. The following two theorems show that axes of certain related points play interesting roles.

THEOREM 3.8. The $\infty^{2}$ axes $l_{T}$ of the points $T$ of a fixed plane $\pi$ are the $\infty^{2}$ chords of the twisted cubic of poles of $\pi$ in a $P^{3}{ }_{40}$ system.

Proof. Any point $T$ of $\pi$ can serve as one of the points whose polar planes provide an axial pencil for the generation of the twisted cubic. By well-known theorems on such generation (5, p. 170), we know that the axis $l_{T}$ is a chord of the twisted cubic. Since this is so for all points of $\pi$, our theorem is established.

THEOREM 3.9. If $l$ is a fixed line, the polar lines $l^{\prime}{ }_{i}$ of $l$ with respect to the pencil of polarities, and the axes $l_{P}$ of the different points $P$ of $l$ form the two systems of generators of a quadric, which (in general) passes through the vertices of the self-polar tetrahedron. 
Proof. Take two points, $Q$ and $R$, arbitrarily on $l$. Their polar planes, with respect to a polarity of the pencil, intersect in the polar line $l^{\prime}{ }_{1}$, with respect to this polarity. As the polarity varies in the pencil the polar planes of $Q$ and $R$ describe projective pencils with axes $l_{Q}$ and $l_{R}$. The lines of intersection $l^{\prime}{ }_{i}$ of corresponding planes describe a regulus, and the axes $l_{Q}$ and $l_{R}$ belong to the conjugate regulus (10, p. 299).

Now we show that the regulus passes through points $A, B, C, D$. Let $l \cdot \alpha=A_{l}$ and $l \cdot \delta=D_{l}$. The polar of $D_{l}$ in plane $\delta$ varies in a pencil about a point, say $D^{\prime}$, as the polarity varies; and, in every polarity, the polar planes of $A_{l}$ pass through $A$. Therefore, in plane $\delta$, the points of the regulus belong to a conic, which can be thought of as the intersection of corresponding lines of two projective pencils of lines through $D^{\prime}$ and $A$. Hence, $A$, and similarly $B$, $C$, and $D$ lie on the regulus.

This construction in plane $\delta$ breaks down in the $P^{3}{ }_{43}$ case, where the polarity induced in one of the faces, say $\delta$, is the same for the entire pencil. In this case, the regulus degenerates.

The first part of the proof meets with a special case in the event that $l$ itself is an axis of a point, say $P$. In this case the regulus degenerates into a cone with vertex $P$.

\section{Tetrahedral pencils of quadrics.}

THEOREM 4.1. Every pencil of polarities determines a pencil of quadrics.

Proof. Consider the polarities $(A B C D)(P \pi)$, with $\pi$ varying through line $p^{\prime}=l_{P}$. Since one possible position for $\pi$ is $P_{p^{\prime}}$, one of the polarities determines a quadric that touches $P_{p^{\prime}}$ at $P$. In fact, every point $X$ lies on such a quadric (touching $X x^{\prime}$ at $X$ ).

Suppose two of the quadrics have a point $R$ in common. Then all the quadrics have $R$ in common.

COROLlary. There is only one quadric through any point which does not lie on the intersection of two.

The points which are common to all the quadrics of the pencil are simply the points which make up the curve of intersection of the "two." This curve we call $\Gamma$, the base curve, of the pencil of quadrics. Since two members of the pencil meet a general plane in a conic, and two conics intersect in four points (in general), the base curve is a quartic. It is clear that a pencil of quadrics is cut by a plane in a pencil of conics.

Now if $R$ (on $\Gamma$ ) is on $A B$, then we are led to one of our cases $P^{3}{ }_{41}, P^{3}{ }_{42}$, or $P^{3}{ }_{43}$. In the simplest case, we have self-dual systems determined in $\delta$ and $\gamma$ with lines $D R$ and $C R$ as common tangents. Therefore, plane $D C R$ is a common tangent plane (at $R$ ) for all quadrics of the pencil. Further, let $Q$ be on $A B$ such that $\mathrm{H}(A B, Q R)$. Then $D C R$ is also a common tangent plane (at $Q$ ). So, we have a $P^{3}{ }_{41}$ system yielding a double-contact of pencil quadrics. 
Suppose in addition to having $R$ (and hence $Q$ ) in common, the quadrics also have a point $T$ in common. Three significant cases arise:

(a) $T$ does not lie on any of the faces of $A B C D$. Since plane $Q R T$ cuts each of the quadrics in a conic, and since a unique conic is determined by the points $Q, R, T$ (we have the tangents at $Q$ and $R$ ), every member of the pencil passes through this conic.

Let plane $\eta$ be the harmonic conjugate of $A B T(=Q R T)$ with respect to $\gamma$ and $\delta$. Then by well-known properties of quadrics, there is a point $U=\eta \cdot C T$ such that $U$ is also a common point of all the quadrics. Therefore, there is another conic determined by $Q, R, U$ (and the tangents at $Q$ and $R$ ), which is common to all the quadrics of the pencil.

Conclusion. This is our $P^{3}{ }_{41}$ case. $\Gamma$ breaks up into two conics which intersect in $R$ and $Q$. The two conics lie in planes which are harmonic conjugates with respect to $\gamma$ and $\delta$.

(b) $T$ is on $C D$. $U$, the harmonic conjugate of $T$ with respect to $C$ and $D$, is also a point common to all the quadrics. Now $Q T$ and $R T$ touch all the quadrics at $T ; Q U$ and $R U$ touch all the quadrics at $U$. Since $Q$ and $R$ are also on the quadrics, we have lines $Q T, R T, Q U$, and $R U$ as common generators for all the members of the pencil.

Conclusion. In this case, $P^{3}{ }_{42}, \Gamma$ is the skew quadrilateral $Q T R U$.

(c) $T$ is in plane $\delta$. Again we have the intersection of $\delta$ with the pencil of quadrics being a single conic through $Q, R$, and $T$. That is, the plane $\delta$ has the same polarity induced in it for all members of the pencil. This corresponds to the $P^{3}{ }_{43}$ system. The infinity of lines joining $D$ to the conic in $\delta$ are tangents to all the quadrics. That is, the pencil has ring contact on this conic.

Conclusion. $A P^{3}{ }_{43}$ system yields a pencil of quadrics which have ring-contact in plane $\delta . \Gamma$ consists of the conic in $\delta$ counted twice. At each point of this conic, the quadrics of the pencil touch.

Another way of arriving at this type of system is to suppose simply that two quadrics of the pencil have a point $R$ in common, with $R$ lying in $\delta$ but not on any edge of $A B C D$. Then $T$, the harmonic conjugate of $R$ with respect to $C$ and $C R \cdot A B$, is also common to the system. Also $U$, the harmonic conjugate of $T$ with respect to $B$ and $B T \cdot A C$, is common; and $V$, the harmonic conjugate of $U$ with respect to $C$ and $C U \cdot A B$, is common; and $W$, the harmonic conjugate of $T$ with respect to $A$ and $B C \cdot A T$, is common. These points, $R, T, U$, $V, W$ determine a conic which is the intersection of $\delta$ with all the quadrics of the pencil.

Suppose that we now begin again with the assumption that two (hence all) of the quadrics have in common a point $Q$, which does not lie on any face of $A B C D$. Then $R$, the harmonic conjugate of $Q$ with respect to $C$ and $C Q \cdot \gamma$, is common to all the quadrics; point $S$, the harmonic conjugate of $Q$ with respect to $D$ and $D Q \cdot \delta$, is common to all the quadrics; finally $T$, the harmonic conjugate of $R$ with respect to $D$ and $D R \cdot \delta$, is common to all the quadrics. Now 
$Q, R, S, T$ are coplanar, so if we assume that the quadrics have another point in common in plane $Q R S T$, we arrive at a previously discussed case, namely $P^{3}{ }_{41}$. Therefore, we assume that the quadrics pass through a point $U$ not in plane $Q R S T$. By the same method used in finding $R, S, T$, we find $V, W, Y$ coplanar with $U$, such that $V, W, Y$ are also common to all the quadrics. Hence, we have eight points which determine a quartic, the curve of intersection of the quadrics. This method of finding $\Gamma$ breaks down if the eight points are associated; for then, any quartic through seven of the points passes through the eighth. In this event we can, by the above method, find many more points belonging to $\Gamma$. Since the "eighth associated point" is unique, we have the opportunity of choosing the eighth point (which finally determines $\Gamma$ ) so that it is not associated.

It can be noted that $\Gamma$ cannot pass through $C$ or $D$, for these points lie in plane $Q R S T$. Likewise, the quartic cannot pass through $A$ or $B$.

Conclusion. This, the most general case, is our $P^{3}{ }_{40}$. It yields a pencil of quadrics whose intersection $\Gamma$ is a quartic which has no multiple points and does not pass through any of the vertices of the fixed self polar tetrahedron.

Summary. The investigation of tetrahedral pencils of polarities has shown that there are four different types, each yielding a distinct pencil of quadrics. Thus, four of the thirteen non-degenerate pencils of quadrics are reached in this manner. The corresponding Segre symbols (6, pp. 304-308; 9, pp. 190-195) are as follows:

$$
P^{3}{ }_{40}-[1111], P^{3}{ }_{41}-[(11) 11], P^{3}{ }_{42}-[(11)(11)], P^{3}{ }_{43}-[(111) 1] .
$$

5. Hexagonal pencils of polarities. Given a self-polar skew hexagon $A P B Q C R$, a polarity is determined (7; pp. 269-288) by the following correspondence of points and planes:

$$
\begin{aligned}
& A \leftrightarrow B Q C, \\
& P \leftrightarrow Q C R, \\
& B \leftrightarrow A R C, \\
& Q \leftrightarrow P A R, \\
& C \leftrightarrow A P B, \\
& R \leftrightarrow P B Q,
\end{aligned}
$$

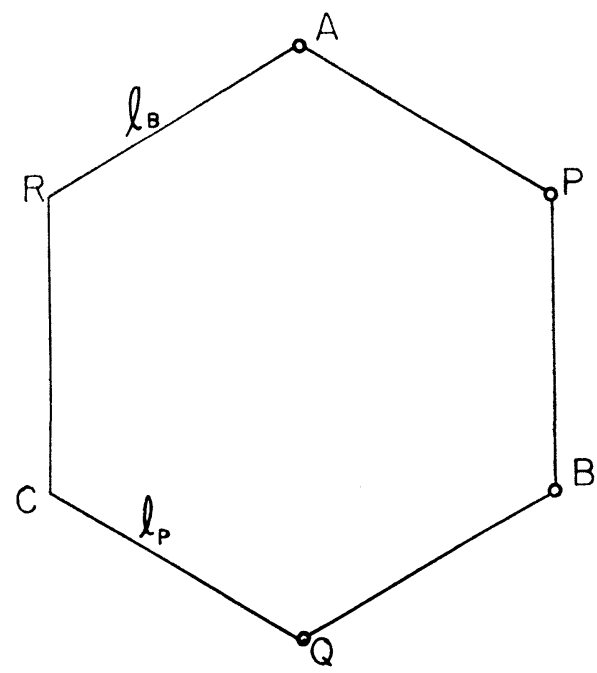

Figure 8 
Definition. If line $R C$ (Fig. 8) is permitted to vary in a regulus such that $R$ varies along a fixed line through $A$, while $C$ varies along a fixed line through $Q$, then we have $\infty^{1}$ polarities determined. We call these a hexagonal pencil of polarities.

The motivation for this definition follows. The main consideration is to arrive at a definition which generalizes the notion of pencils of polarities as previously defined in $S_{3}$. Then suppose we were to let a point, say $R$, vary. It would have to vary in a twisted cubic, a conic, a line; for these are the loci of poles of a fixed plane $P B Q$ in a pencil of polarities. This sort of definition is, of course, unsuitable. We seek, then, an analogue of the two-dimensional case, and thus consider a line varying. Since its polar line (the opposite side of the hexagon) remains fixed, Theorem 3.9 indicates that our line should vary in a regulus.

Thus we let $R C$ vary in a regulus. Does this mean that lines $A R$ and $C Q$ must also vary? The answer to this question is no; for $A R$ and $C Q$ are the axes, $l_{B}$ and $l_{P}$, of the points $B$ and $P$ (respectively), and therefore must be members of the associated regulus. If then $A R$ and $C Q$ were variable, different complementary generators would be intersecting in $A$ and in $Q$. This situation would always lead to a degenerate regulus.

Therefore, in order to characterize a hexagonal pencil of polarities, we must allow line $R C$ to vary in a regulus, with $R$ always on the fixed line $l_{B}=A R$, and $C$ always on the fixed line $l_{P}=Q C$.

Define $\alpha=B Q C$, and let $A P$ meet $\alpha$ in $D$. Join $A Q$ and $D Q$ (Fig. 9). The polar plane of $Q$ is $\xi=P A R$. Plane $\xi$ cuts $\alpha$ in $D E$, where $E=C Q \cdot \xi$. This leads to a self-polar tetrahedron $A Q D E$, which is fixed for the entire pencil of polarities.

Now for a point not on the tetrahedron and its polar plane. Consider the point $M=P B \cdot A R Q$. Its polar plane is $\mu=R C(B Q \cdot \xi)$. Call $F$ the point determined by $B Q \cdot \xi$. Then $\mu=R C F$. By Theorem 3.9 we know that $D$ also

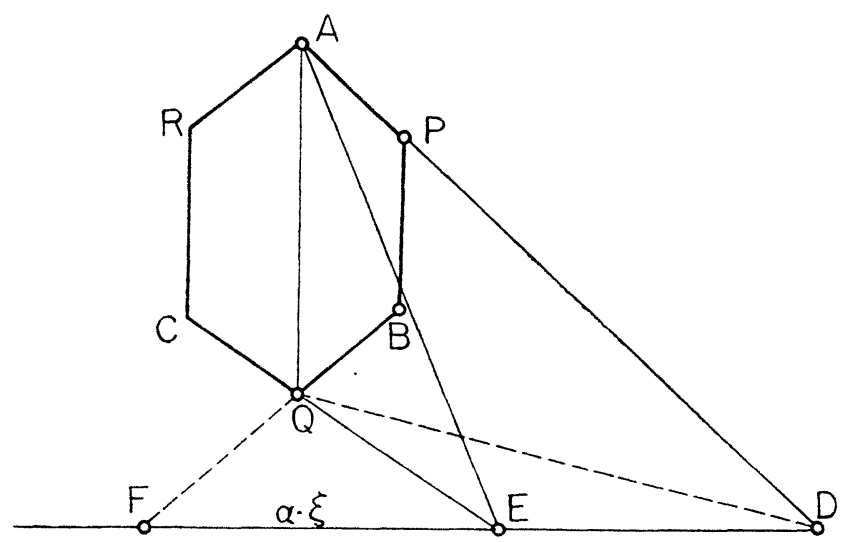

Figure 9 
lies on the regulus $(R C)$, with the notable exception of the $P^{3}{ }_{43}$ case. Hence, in general, $D E$ is a generator of the conjugate regulus. Therefore, $F$ in general lies on the quadric.

We wish to show that $\mu$ varies in an axial pencil. A projective correspondence exists between the axial pencil $C Q$ and the planes $\mu$, with corresponding planes intersecting in $R C$. Thus, there is a line $l_{M}-$ through $F$ and lying in the quadric - which is the axis of the pencil of planes $\mu$.

Now, from Theorem 3.1 we know that the polar planes of any fixed point vary in an axial pencil. In this case, where we have a fixed self-polar tetrahedron, we can show that each axis passes through $D E$. For the pencil of polarities induces a self-dual system in plane $Q D E$, and the properties of selfdual systems indicate that the polars of any fixed point vary in a pencil whose vertex is always on the same side of the fixed self-polar triangle. Hence, in general, the axes of the $\infty^{3}$ points of $S_{3}$ form a linear complex of lines through $D E$.

According as the axis of $\mu$ passes through one, two, or three edges of $A Q D E$, we get cases $P^{3}{ }_{41}, P^{3}{ }_{42}$, or $P^{3}{ }_{43}$. But it appears as though we can never arrive at $P^{3}{ }_{40}$. (Note that in the case $P^{3}{ }_{43}$, we get one of either $R$ or $C$ fixed for the pencil. The regulus $(R C)$ degenerates into a plane pencil of lines the vertex of which is the fixed point $R$ or $C$, as the case may be.)

Again, by methods of $\S 4$, we can show that a pencil of polarities leads to a system of quadrics, which we call a pencil of quadrics.

We know that the hexagon will yield pencils of quadrics $P^{3}{ }_{4 k}(k=1,2,3)$, but the important question to answer is: Do the hexagonal pencils yield any systems of quadrics which do not have fixed self-polar tetrahedra?

In order to find the answer to such a question, we degenerate the self-polar tetrahedron which appears in our hexagonal development. The tetrahedron is $A Q D E$. The various ways in which it may degenerate are listed as follows:

$5.1 D$ lies on plane $A Q E$.

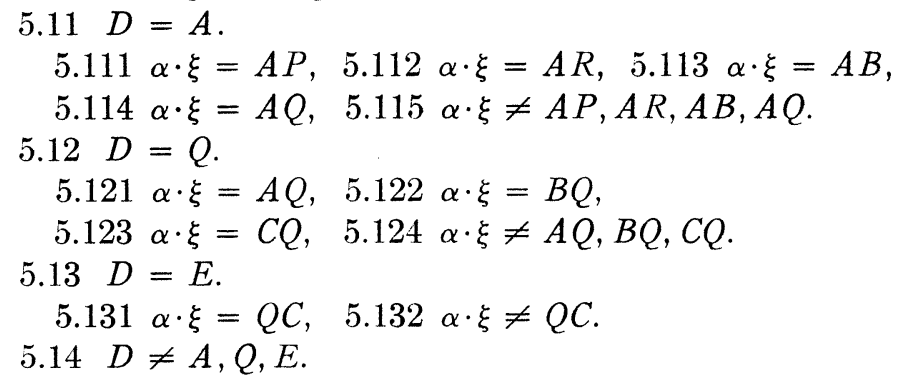

5.2. Elies on plane $A D Q$.

$5.21 E=A, 5.22 E=Q, 5.23 E=D, \quad 5.24 E \neq A, Q, D$.

5.3 Q lies on plane $A D E$.

5.4 A lies on plane $Q D E$.

$5.41 A=Q, 5.42 A=D, \quad 5.43 A=E, \quad 5.44 A \neq Q, D, E$. 
We proceed to investigate these cases.

5.111 Here $A, P, B, Q, C$ are coplanar (all on $\alpha$ ). This case degenerates completely.

$5.112 R$ on $\alpha$ implies that $\alpha$ is the polar plane of $P$ as well as of $A$. Impossible.

5.113 Since $\alpha \cdot \xi$ is the polar line of $A Q$, the polar plane of $B$ (on $\alpha \cdot \xi$ ) always passes through $Q$ as well as $A, R$, and $C$. Hence $P$ and $B$ have the same polar plane. Therefore this case degenerates completely.

5.114 (Fig. 10). Since $\alpha$ is a common tangent plane at $A$ for all the quadrics (if the pencil exists), they have contact of order 1 or higher. Actually, they must have four-point contact (third order). For consider the polarities induced in plane $B A R$. The conics all touch line $A B=a$ at $A$. Any fixed point on $A R$ has a variable polar through $B$. This rules out the possibility of constructing a self-polar triangle. Thus two-point contact is ruled out. Since $B$ has a fixed polar line $A R$, three-point contact is ruled out $(\mathbf{1 0}, \mathrm{p} .75)$. Thus the conics must have four-point contact at $A$; and therefore, the quadrics have fourpoint contact at $A$. Similarly for $Q$.

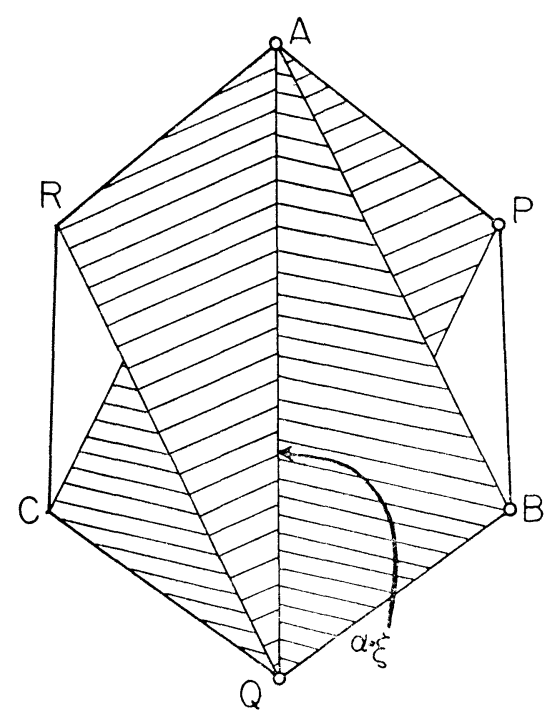

FigURE 10

Now we look at plane $A P B$. The pencil of conics in this plane must also have four-point contact at $A$. Again, using a result of Veblen and Young (10, p. 75), $B$ has a fixed polar $b$ through $A$. But $b=A R C \cdot A P B$. Therefore $C$ must be fixed on $Q C$. Likewise we can show that $R$ is fixed on $A R$. Impossible. 
5.115 Let $E^{\prime}=\xi \cdot Q B$ (Fig. 11).

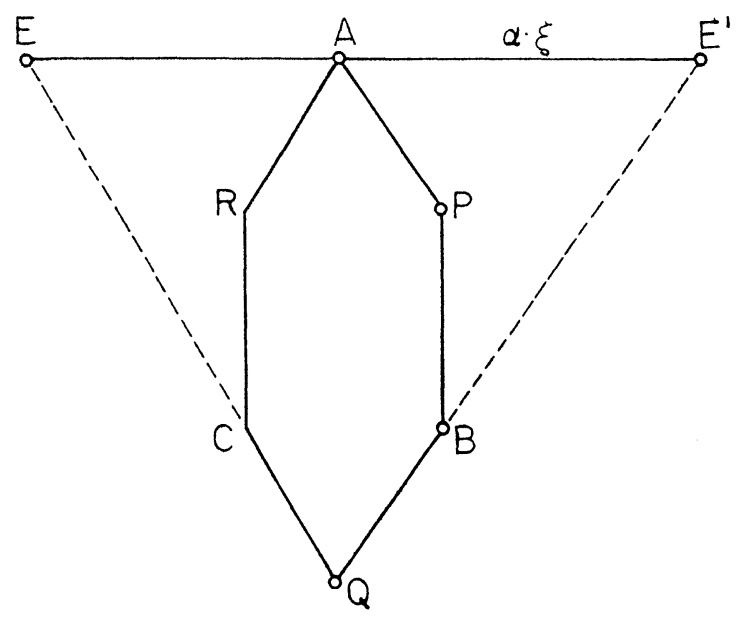

FIGURE 11

5.1151 Suppose there is, in the plane $\alpha$, a point $X$, distinct from $A$, such that $X$ is common to the quadrics of the pencil (if it exists). Since the polarity in $\alpha$ is degenerate, the line $x$ (the polar of $X$ ) must be $A X$.

Since the polar of any point on $\alpha \cdot \xi$ is $A Q, X$ does not lie on $A Q$. (The situation under discussion may arise when the third generator of the regulus $(R C)$ lies in $\alpha$. This implies that $C$ remains fixed on the intersection of the third generator and $Q C$.) If we wish $C$ to be our point $X$, or any point which is self-conjugate for all the polarities, we let $C$ be on $A B$ (Fig. 12). $C$ is on plane $A P B$ for every polarity since it is self-conjugate. Let line $l$ be the harmonic conjugate of line $A B$ with respect to $A C$ and $\alpha \cdot \xi$. Then $X_{1}=E^{\prime} C \cdot l$ is also on all the quadrics. Likewise, $X_{2}=X_{1} E \cdot A B$ is on all the quadrics.

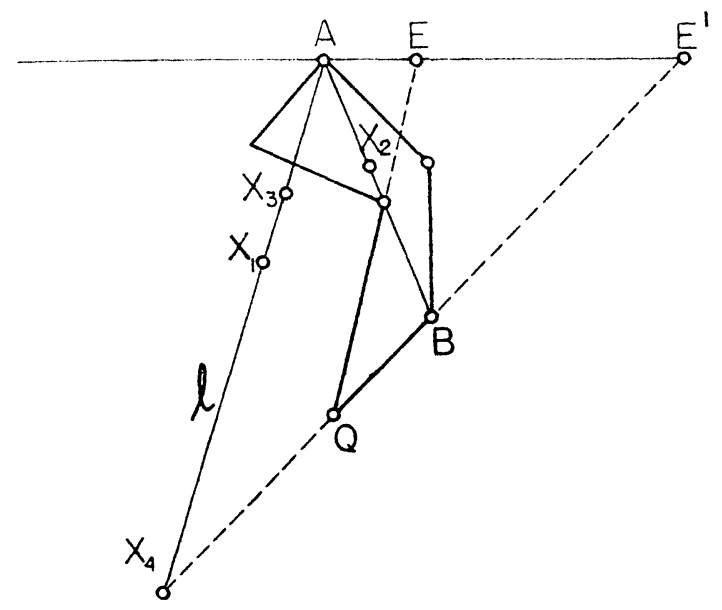

Figure 12 
Also, $X_{3}=E^{\prime} X_{2} \cdot l$. Moreover, the lines $A X_{2}$ and $A X_{3}$ are common to all the quadrics. $\alpha$ is the common tangent plane at $A, A P B$ is the common tangent plane at $C, A C R$ is the common tangent plane at $B . A, B$, and $C$ are, therefore, double points of the curve of intersection $\Gamma$. Actually, the entire line $A B$ is a line of double points.

Now $X_{4}=l \cdot B Q$ has as its polar the plane $A R X_{4}$, and $X_{5}=l \cdot Q C$ has $A P X_{5}$ as its polar plane. Further, these planes are common tangent planes for the pencil of quadrics at points $X_{4}$ and $X_{5}$, respectively. Hence, line $l$ is also a line consisting of double points of $\Gamma$.

Conclusion. We have arrived at a pencil of quadrics whose curve of intersection $\Gamma$ is a pair of intersecting lines counted twice. All of the quadrics touch at each point of the two lines. We call this a $P^{3}{ }_{61}$ system.

5.1152 Suppose $X$ is on $\Gamma$ (that is, is common to all the quadrics) and on $\xi$, and that $X$ lies on neither line $A R$ nor line $A P$ (Fig. 13). Let $U=E X \cdot A P$. Call $X_{1}$ the harmonic conjugate of $X$ with respect to $E$ and $U$. Let $V=A R$. $E X_{1}$. Call $X_{2}$ the harmonic conjugate of $X_{1}$ with respect to $E$ and $V . X_{1}$ and $X_{2}$ are points of $\Gamma$, too. A unique conic $\mathscr{C}$ is determined in plane $\xi$ by $X, X_{1}$, $X_{2}$ and $A$ (the tangent at $A$ is $\alpha \cdot \xi$ ). Hence, the polarity in plane $\xi$ is the same for all the polarities of the pencil. Further, the lines joining $Q$ to the conic $\mathscr{C}$ are tangents to all the quadrics. Therefore this system (if it exists) is a ringcontact system, the same as $P^{3}{ }_{43}$.

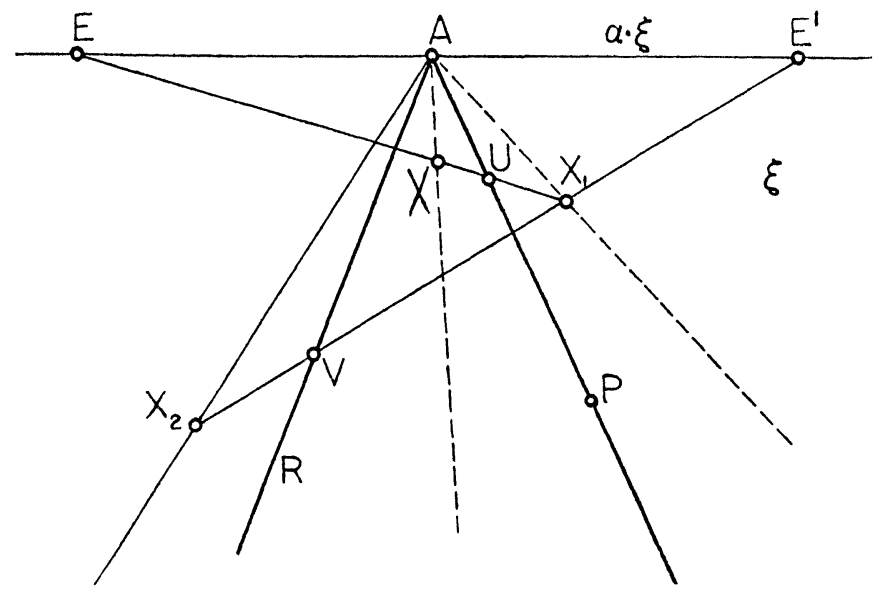

FIGURE 13

5.1153 We now allow a point of $\Gamma$ to be on $A P$ or $A R$. By assuming $X$ on $\Gamma$ to be on $A P$ and taking harmonic conjugates as done above we get two lines of points which belong to $\Gamma$. They are all double points because the lines from $Q$ to these lines are all tangents. Hence, all the quadrics touch on our two lines of $\Gamma$. This is a ring-contact system of quadrics touching at a degenerate conic. It reduces to our $P^{3}{ }_{61}$ case. 
5.1154 Suppose $X$ is on $\Gamma$, not on $\xi$, not on $A Q P$, and not on $A Q R$. For all the polarities of the pencil the polar plane of $E^{\prime}$ is $\eta^{\prime}=A Q R$ and the polar plane of $E$ is $\eta=A Q P$. Let $X_{1}$ be such that $\mathrm{H}\left(E(E X \cdot \eta), X X_{1}\right)$. Then $X_{1}$ is on $\Gamma$. Let $X_{2}$ be such that $\mathrm{H}\left(E^{\prime}\left(E^{\prime} X \cdot \eta^{\prime}\right), X X_{2}\right)$. The point $A$ (with its tangent $\alpha \cdot \xi), X, X_{1}, X_{2}$ determine a conic $\mathscr{C}_{1}$ in plane $E E^{\prime} X . \mathscr{C}_{1}$ is part of $\Gamma$. (Additional points of $\mathscr{C}_{1}$ may be found by repeating above method.) Consider plane $\theta$, the harmonic conjugate of $E E^{\prime} X$ with respect to $\alpha$ and $\xi$. The projection of $\mathscr{C}_{1}$ on $\theta$ through point $Q$ is a conic $\mathscr{C}_{2}$, which also belongs to $\Gamma$. Hence $\Gamma$ consists of two conics which have $\alpha \cdot \xi$ as a common tangent at point $A$. We call this a $P^{3}{ }_{62}$ system.

5.1155 If we consider the previous case without the assumption that $X$ is not on $\eta$ and not on $\eta^{\prime}$, we are led to a degenerate conic, consisting of two lines meeting in $A$, in plane $E E^{\prime} X$; and in plane $\theta$ we also have such a conic. $\Gamma$ here would consist of four lines all intersecting in $A$. This is not possible as the intersection of two non-degenerate quadrics. Hence this case does not exist.

5.121 (Fig. 14) $D=Q$ implies that $A, \mathrm{P}$ and $Q$ are collinear and $\alpha \cdot \xi=A P$. Therefore the polar line of $\alpha \cdot \xi$ is $Q C$. But $\alpha \cdot \xi$ is its own polar line. Therefore $Q C=\alpha \cdot \xi$. For every polarity of the pencil, the polar plane of $B$ is $A R C=\xi$. Hence $B$ and $Q$ always have the same polar plane, which is impossible.

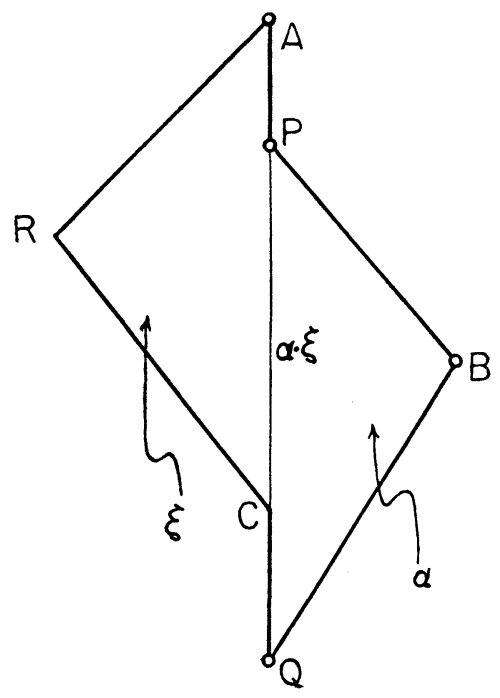

Figure 14

$5.122 \alpha \cdot \xi=Q B$ is the polar line of $A R$ and $A Q$. Therefore $A R=A Q$. This case degenerates with $A, R, P, B, Q$ all on $\xi$, and points $B$ and $Q$ having the same polar plane. 
$5.123 \alpha \cdot \xi$ is the polar line of $A Q$; hence $C Q=\alpha \cdot \xi$ has $A P=A Q$ as its polar line. $A, R, P, C, Q$ are all on $\xi$. This case degenerates.

$5.124 \alpha \cdot \xi$ is not any of $A Q, B P, Q B, C Q$. The polar line of $A Q$ is $\alpha \cdot \xi$. But $A Q=A P$ (since $D=A P \cdot \alpha$ ), and the polar line of $A P$ is $C Q$. Therefore, $\alpha \cdot \xi=C Q$. This contradicts the hypothesis of this case.

$5.131 Q C$ is the polar line of $A P$, and $\alpha \cdot \xi$ is the polar line of $A Q$. Therefore $A Q=A P$ and $Q=D=E$. This reduces to case 5.123.

5.132 In this case we can find a fixed self-polar tetrahedron $A D^{\prime} E^{\prime} Q$, where $E^{\prime}=B Q \cdot \xi$ and $D^{\prime}=A R \cdot \alpha$. This reduces to previously discussed cases (Fig. 15).

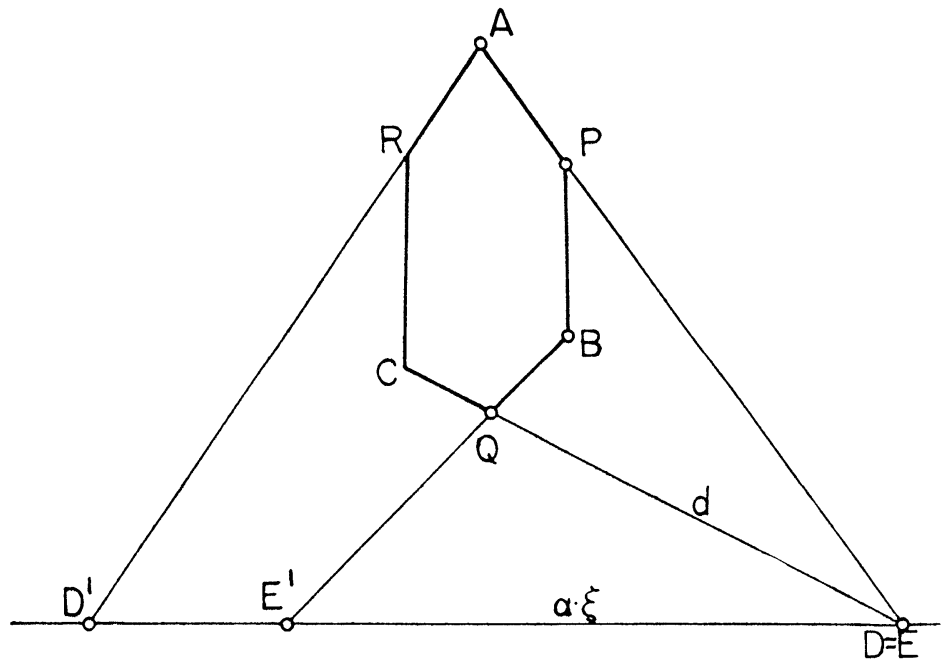

FIGURE 15

However, we may attempt to degenerate $A D^{\prime} E^{\prime} Q$ by allowing $D^{\prime}=E^{\prime}$. The polar plane of $D$ is $A P Q$ for every polarity. Therefore, $D$ is self-conjugate in every polarity. Similarly, the polar plane of $D^{\prime}$ is $A Q B$ and $D^{\prime}$ is selfconjugate in every polarity. If we now examine the polarity induced in $\alpha$, we see that $D \leftrightarrow Q D$ and $D^{\prime} \leftrightarrow Q D^{\prime}$. Hence $Q \leftrightarrow \alpha \cdot \xi$. Hence, we have a selfdual system in $\alpha$. The self-polar triangles are formed by $Q$ and any pair of the involution $\left(D^{\prime} D^{\prime}\right)(D D)$ on $\alpha \cdot \xi$. We can find infinitely many self-polar tetrahedra which are fixed for the pencil by taking $A, Q$, and a pair of the indicated involution on $\alpha \cdot \xi$. These cases have already been discussed in $\S 3$ and $\$ 4$.

$5.14 D$ lies on $A Q E . D \neq A, Q, E . Q C \cdot \xi=E$ and $D$ is on $\alpha \cdot \xi$. But $D$ is on $A Q E$; hence $D$ is on $Q E$. Therefore $D=E$, which contradicts the hypothesis.

5.21 (Fig. 16). $E=A$ implies $A$ is on $Q C$. The polar plane of $P$ is $R C Q$, and the polar plane of $B$ is $A R C=R C Q$. Two distinct points have the same polar plane which is impossible. 


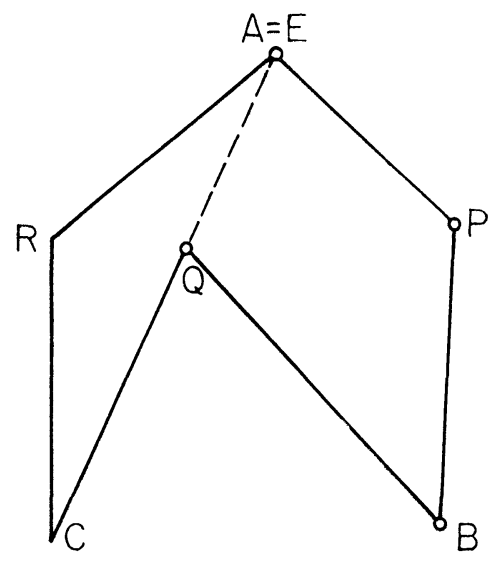

FIGURE 16

5.22 This is the same case as 5.11 with the letters $D$ and $E$ interchanged and the letters $A$ and $Q$ interchanged.

5.23 Although tetrahedron $A Q E D$ breaks down, we can find another selfpolar tetrahedron $A Q D^{\prime} E^{\prime}$. We can proceed as in 5.132 with the same results.

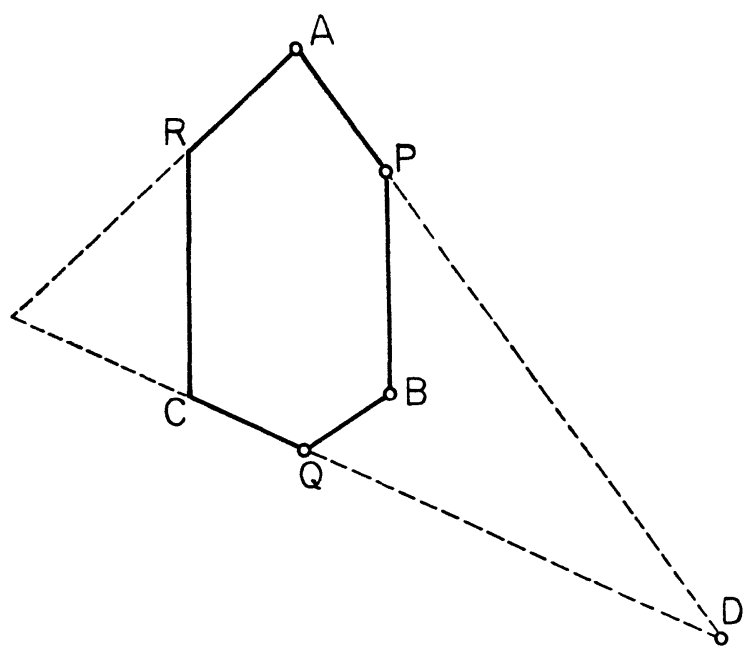

FIGURE 17

5.24 (Fig. 17) $E$ on plane $A D Q$ implies that $D$ is on line $Q C$. Hence, $A P$ and $Q C$ are coplanar. $E \neq D$, and $E D$ is $\alpha \cdot \xi=Q C$. $A, P, Q, C, R$ are all on $\xi$. This case is degenerate.

5.3 $Q$ lies on $A D E$. This is essentially the same as 5.1 and 5.2 where $E$ lies on $A D Q$, except in the case where $Q=A$. But in this case $\alpha=\xi$, and all the points are coplanar. 
$5.41 A$ lies on $Q D E$ and $A=Q$. This degenerates; for $A=Q$ implies $\alpha=\xi$, and this implies that all the points are coplanar.

5.42 This case was treated in 5.11 .

5.43 This case was treated in 5.21 .

$5.44 Q D E=\alpha$. Hence, $A$ is on $\alpha . A P \cdot \alpha=D$ implies $A=D$, which case has already been treated.

Summary. The development of hexagonal pencils of polarities has shown them to be not quite as good as a generalization as pentagonal pencils were in $S_{2}$. The hexagonal pencils have yielded two pencils of quadrics which tetrahedral pencils failed to produce. However, one of the systems of quadrics $\left(P^{3}{ }_{40}\right)$ which arose from the tetrahedral development cannot be reached as a system of the hexagonal type.

Below is a listing of all the systems of quadrics by means of their Segre symbols matched with their pencils of polarities symbols when such exist.

\begin{tabular}{|c|c|}
\hline$\left[\begin{array}{llll}1 & 1 & 1 & 1\end{array}\right]-P_{40}^{3}$ & {$\left[\left(\begin{array}{ll}2 & 1) 1 \\
& 1]-P^{3}{ }_{62}\end{array}\right.\right.$} \\
\hline 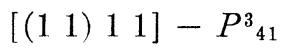 & {$\left[\left(\begin{array}{lll}2 & 1 & 1\end{array}\right)\right]-P_{61}^{3}$} \\
\hline$\left[\left(\begin{array}{lll}1 & 1\end{array}\right)\left(\begin{array}{ll}1 & 1\end{array}\right)\right]-P_{42}^{3}$ & {$\left[\begin{array}{ll}2 & 2\end{array}\right]$} \\
\hline$\left[\left(\begin{array}{lll}1 & 1 & 1\end{array}\right) 1\right]-P_{43}^{3}$ & {$\left[\left(\begin{array}{ll}2 & 2\end{array}\right)\right]$} \\
\hline$\left[\begin{array}{lll}2 & 1 & 1\end{array}\right]$ & {$\left[\begin{array}{ll}3 & 1\end{array}\right]$} \\
\hline$\left.\left[\begin{array}{lll}2 & (1 & 1\end{array}\right)\right]$ & $\begin{array}{l}\left.\left[\begin{array}{ll}(3 & 1\end{array}\right)\right] \\
{[4]}\end{array}$ \\
\hline
\end{tabular}

6. Pencils of polarities in $S_{n}$. A symmetric (i.e., non-null) polarity in $S_{n}$ is determined by a self-polar simplex $\Sigma_{n}=A_{1} A_{2} \ldots A_{n+1}$ and a pair of corresponding elements: point $P$ and the $(n-1)$-flat (prime) $\pi$. The notation for this polarity is the usual $\left(A_{1} A_{2} \ldots A_{n+1}\right)(P \pi)$.

In general, we shall denote the prime $A_{1} A_{2} \ldots A_{i-1} A_{i+1} \ldots A_{n+1}$ by the symbol $\alpha_{i} ; \alpha_{i}$ is then the polar prime of $A_{i}$.

Definition. If $\pi$ varies in a pencil about the $(n-2)$-flat $p$, we have $\infty 1$ polarities $\left(A_{1} A_{2} \ldots A_{n+1}\right)(P \pi)$ determined. We call these a pencil of polarities in $S_{n}$.

THeORem 6.1. The polar prime $\chi$ of any fixed point $X$, with respect to a pencil of polarities, varies in a pencil about an $(n-2)$-flat.

Before embarking on a proof we must consider the problem of constructing (4) the polar prime of an arbitrary point $X$. Such a construction can be carried out in $S_{2}$ and $S_{3}$. We assume that it can also be done in $S_{n-1}$. Let

$$
A_{i} P \cdot \alpha_{i}=P_{i}, A_{i} X \cdot \alpha_{i}=X_{i}, \pi \cdot \alpha_{i}=p_{i}, \quad i=1,2, \ldots, n
$$


$A_{1} P$ is the polar line of the $(n-2)$-flat $p_{1}$. Every point on $A_{1} P$ is conjugate to $p_{1}$. In particular, $P_{1}$ is such a point. Hence, we have a polarity induced in the prime $\alpha_{1}$. By the induction hypothesis, we can find the polar $(n-2)$ flat of point $X_{1}$. Call it $x_{1}$. We repeat this construction in prime $\alpha_{2}$ arriving at an $(n-2)$-flat $x_{2}$ which meets $x_{1} . \quad \chi$, the polar prime of $X$, must pass through $x_{1}$ and $x_{2}$, for $X$ is conjugate to every point on $x_{1}$ and to every point on $x_{2}$. Since these two $(n-2)$-flats meet, they determine a prime which is in fact $\chi$, the polar prime of point $X$.

Proof of Theorem 6.1. We proceed by induction. The theorem is true for $n=2,3$. Suppose it is also true for $S_{n-1}$.

Let $\pi$ vary about $p$. Since $p$ cannot lie in more than $n-1$ of the $n+1$ faces of $\Sigma_{n}$, we suppose that $\alpha_{1}$ and $\alpha_{2}$ are two in which $p$ does not lie. Then $p \neq p_{1}=\pi \cdot \alpha_{1} ; p_{1}$ varies in a pencil about an $(n-3)$-flat in face $\alpha_{1}$. By the induction hypothesis, $x_{1}$ likewise varies in a pencil (in $\left.\alpha_{1}\right)$ about an $(n-3)$ flat $x^{\prime}{ }_{1}$. Similarly, $x_{2}=\chi \cdot \alpha_{2}$ varies in a pencil (in $\left.\alpha_{2}\right)$ about an $(n-3)$-flat which we call $x_{2}^{\prime}$. In each polarity of the pencil we have $\chi$ determined by $x_{1} \cdot x_{2} . \chi$ must pass through $x_{1}^{\prime} \cdot x^{\prime}{ }_{2}$ in each of the polarities. Hence, the polar prime $\chi$ of point $X$ varies in a pencil about $x^{\prime}{ }_{1} \cdot x^{\prime}{ }_{2}$.

In case $x_{1}^{\prime}=x_{2}^{\prime}$, then we can take $x_{3}^{\prime}, x_{4}^{\prime}, \ldots, x_{n+1}^{\prime}$. They cannot all be equal.

In $S_{n}$ there are for consideration the cases $P_{n+1, k}^{n}$ where $k=0,1,2, \ldots$, $\frac{1}{2} n(n-1)$ according as $p$ meets none, $1,2, \ldots, \frac{1}{2} n(n-1)$ edges of $\Sigma_{n}$. $p$ passes through the maximum number of edges when it lies entirely in a face, thus meeting all of the $\frac{1}{2} n(n-1)$ edges of $\Sigma_{n}$ in the face. It is clear that this is not a complete classification for there may be several distinct pencils in $S_{n}$ with the same $k$ when $n>3$; e.g., in $P^{4}{ }_{53}, p$ meets three edges of $\Sigma_{n}$ but different cases occur when the three edges are coplanar and not.

The $P_{n+1,1}^{n}$ system: In this case we suppose that $p$ meets only one edge, say $A_{1} A_{2}=a$ of $\Sigma_{n}$. Two-dimensional self-dual systems are induced in every plane of $\Sigma_{n}$ which is incident with $A_{1} A_{2}$. Hence, the involution of conjugate points on $A_{1} A_{2}$ is the same for all the polarities. Therefore, any pair of the involution on $A_{1} A_{2}$ together with the points $A_{3}, A_{4}, \ldots, A_{n+1}$ form a fixed self-polar $n$-simplex for the pencil of polarities. Hence

Theorem 6.2. A $P_{n+1,1}^{n}$ system has $\infty^{1}$ self-polar $n$-simplexes, each one of which serves for the entire pencil.

Reasoning analogous to that of Theorem 3.2 gives us

THEOREM 6.3. The product of two polarities belonging to the same $P_{n+1,1}^{n}$ system is a general axial homography (a collineation which leaves invariant every point on a line and every prime on an $(n-2)$-flat). 
Proceeding similarly, we have

THEOREM 6.4 .

(a) The $P_{n+1, k}^{n}$ system has $\infty^{k}$ self-polar $n$-simplexes, each of which serves for the entire pencil.

(b) If two polarities belong to the same $P_{n+1, k}^{n}$ system $(k=2,3,4, \ldots$, $\left.\frac{1}{2} n(n-1)-1\right)$, their product is a k-axial homography (a collineation having $k$ skew lines which are pointwise invariant).

The $P_{n+1, \frac{1}{2} n(n-1)}^{n}$ system is of particular interest since it is the direct generalization of the self-dual system. $p$ now lies entirely in a face, say $\alpha_{1}=A_{2} A_{3} \ldots A_{n+1}$, of $\Sigma_{n}$. The polarity induced in this face is the same for each member of the pencil. The polarity is always $\left(A_{2} A_{3} \ldots A_{n+1}\right)\left(P_{1} p\right)$. Hence the polar primes of point $X$ (for all members of the pencil) pass through $x_{1}$. The involutions induced on all the edges in $\alpha_{1}$ are the same for every polarity in the pencil. By taking any combination of pairs of the involutions on these edges together with the point $A$, we can arrive at $\infty^{\frac{1}{n}(n-1)}$ self-polar simplexes. Hence,

Theorem 6.5. $A P_{n+1, \frac{1}{2} n(n-1)}^{n}$ system admits a prime $\alpha_{1}$, upon which the induced polarity is the same for all members of the pencil. The polars of any point $P$ pass through a fixed $(n-2)$-flat $p$ on prime $\alpha_{1}$, and the poles of any prime $\pi$ form a range of points on a fixed line $A_{1} P_{1}$, where $A_{1}$ is the pole of $\alpha_{1}$ for all the polarities.

THEOREM 6.6. If two polarities belong to the same $P_{n+1, \frac{1}{2} n(n-1)}^{n}$ system, their product is an homology.

Remark. We speak of an homology in $S_{n}$ when the collineation leaves invariant every prime through a point and every point on a prime.

Proof. We suppose that $p$, the axis of $\pi$, lies in $\alpha_{1}$. From the construction of pole and polar prime, we know that every prime through $A_{1}$ is fixed under the product of two polarities, and so is every pencil of primes whose axis lies in $\alpha_{1}$.

Consider any point $X$ in $\alpha_{1}$. Its polar prime $\chi$ intersects $\alpha_{1}$ in the $(n-2)$ flat $x_{1}$. If we operate with another member of the $P_{n+1, \frac{1}{2} n(n-1)}^{n}$ system, we find that $x_{1} \rightarrow X$ because the polarity induced in $\alpha_{1}$ is the same for all members of the pencil of polarities. Hence, $X \rightarrow X$ under the product, which shows that every point of prime $\alpha_{1}$ is invariant.

Now consider an arbitrary prime $\rho$ through $A_{1}$. Let $\rho \cdot \alpha_{1}=r_{1}$. Let $R_{1}$ be the pole of $r_{1}$ in plane $\alpha_{1}$. Then $R$, the pole of prime $\rho$, is on the line $A_{1} R_{1}$. Then operate on $R$ with another member of the system. The construction tells us that $R$ maps into a prime through $r_{1}$. Since every line through $A_{1}$ is invariant, we have the prime $r_{1} A_{1}$ invariant. Hence, the product is an homology. 
THEOREM 6.7. Every homology can be expressed as the product of two polarities belonging to a $P_{n+1, \frac{1}{2} n(n-1)}^{n}$ system.

Proof. The homology whose prime of perspectivity is $\alpha_{1}$ and whose center is $A_{1}$, and with a pair of corresponding points $P_{1}$ and $P_{2}$ (on a line through $\left.A_{1}\right)$ is the product of two polarities $\left(A_{1} A_{2} \ldots A_{n+1}\right)\left(P_{1} \pi\right)$ and $\left(A_{1} A_{2} \ldots A_{n+1}\right)$ $\left(P_{2} \pi\right)$, where $\pi$ is an arbitrary prime.

Theorem 6.8. The locus of poles of a fixed prime in general position with respect to a $P_{n+1,0}^{n}$ system is a rational normal $n$-ic curve.

Proof. Let $\chi$ be a fixed prime. Take $n$ distinct points $Q_{1}, Q_{2}, \ldots, Q_{n}$ on $\chi$. The intersection of the polar primes of these $n$ points is the pole $X$ of $\chi$. The previous theorems indicate that as $\pi$ varies in the pencil the polar primes of $Q_{1}, Q_{2}, \ldots, Q_{n}$ vary in projective axial pencils. The locus, therefore, is a rational normal $n$-ic.

In $P_{n+1, k}^{n}\left(k=1,2,3, \ldots, \frac{1}{2} n(n-1)-1\right)$ the rational normal $n$-ic degenerates into an $(n-k)$-ic or lower order curve which is confined to an $(n-k)$-flat. This follows from reasoning precisely the same as used in the proof of Theorem 3.9. The discussion of the $P_{n+1, \frac{1}{2} n(n-1)}^{n}$ system in Theorem 6.3 indicates that in this case the locus is a line which passes through exactly one vertex $A_{i}$ of $\Sigma_{n}$, where $A_{i}$ is the pole of the face of $\Sigma_{n}$ with the same induced polarity for all members of the pencil.

Proceeding in a manner analogous to that in $\S 3$, we might ask what happens to the $\infty^{n-1}$ axes of $l_{T}$ of the points $T$ on a fixed prime. The general case is most interesting.

THEOREM 6.9. The $\infty^{n-1}$ axes of $L_{T}$ of the points $T$ of a fixed prime $\pi$ are the $\infty^{n-1}$ chords of the rational normal $n$-ic in a $P_{n+1,0}^{n}$ system.

Proof. Any point $T$ of $\pi$ can serve as one of the points whose polar prime provides an axial pencil for the generation of the $n$-ic. By well-known theorems concerned with such a generation, we know that the axis $l_{T}$ is a chord of the $n$-ic. Since this is so for all points of $\pi$, the theorem is established.

For any given $n$, a detailed investigation, analogous to that in $\$ 4$, may be carried out to develop the various pencils of quadrics in $S_{n}$. Such a presentation here would be lengthy and, I fear, uninteresting because of its repetitious nature. However, such would not be the case with a development of pencils of polarities and pencils of quadrics by means of a self-polar $(n+2)$-gon. While it is clear that self-polar $(n+2)$-gons always exist in $S_{n}$, I confess that I have not discovered any general method of investigation of the pencils of polarities which they yield. The method of attack in $S_{3}$ does not readily lend itself to use in higher dimensions; for even in $S_{4}$ we would have an enormous number of special cases to treat. 


\section{REFERENCES}

1. R. Baldus, Zur Klassification der ebenen und räumlichen Kollineationen, Sitz. Bayerischen Akad., 1928, 375-395.

2. H. S. M. Coxeter, The real projective plane (New York, 1949; Cambridge University Press, 1955).

3. _- Projective geometry, Math. Magazine, 23 (1949), 79-97.

4. A. P. Dempster and S. Schuster, Constructions for poles and polars, Pacific J. Math., 5 (1955), 197-199.

5. L. Godeaux and O. Rozet, Leçons de géometrie projective (2nd ed., Liège, 1952).

6. W. V. D. Hodge and D. Pedoe, Methods of algebraic geometry, vol. 2 (Cambridge, 1952).

7. Th. Reye, Ueber Polfünfecke und Polsechsecke raumlicher Polarsysteme, J. reine angew. Math., 77 (1874), 269-288.

8. D. M. Y. Sommerville, An introduction to the geometry of $n$ dimensions (London, 1929).

9. J. A. Todd, Projective and analytical geometry (London, 1947).

10. O. Veblen and J. W. Young, Projective geometry, vol. 1 (Boston, 1910).

11. G. K. C. von Staudt, Geometrie der Lage (Nuremberg, 1847).

\section{Polytechnic Institute of Brooklyn}

